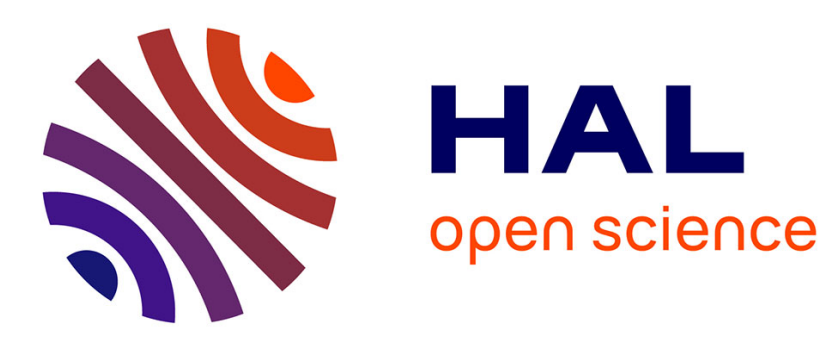

\title{
Moyennes de fonctions arithmétiques de formes binaires
}

Régis de La Bretèche, Gerald Tenenbaum

\section{To cite this version:}

Régis de La Bretèche, Gerald Tenenbaum. Moyennes de fonctions arithmétiques de formes binaires. Mathematika, 2012, 58, pp.290-304. hal-01281335

\section{HAL Id: hal-01281335 \\ https://hal.science/hal-01281335}

Submitted on 2 Mar 2016

HAL is a multi-disciplinary open access archive for the deposit and dissemination of scientific research documents, whether they are published or not. The documents may come from teaching and research institutions in France or abroad, or from public or private research centers.
L'archive ouverte pluridisciplinaire HAL, est destinée au dépôt et à la diffusion de documents scientifiques de niveau recherche, publiés ou non, émanant des établissements d'enseignement et de recherche français ou étrangers, des laboratoires publics ou privés. 


\title{
Moyennes de fonctions arithmétiques de formes binaires
}

\author{
R. de la Bretèche \& G. Tenenbaum
}

\begin{abstract}
Extending classical results of Nair and Tenenbaum, we provide general, sharp upper bounds for sums of the type

$$
\sum_{\substack{u<m \leqslant u+v \\ x<n \leqslant x+y}} F\left(Q_{1}(m, n), \ldots, Q_{k}(m, n)\right)
$$

where $x, y, u, v$ have comparable logarithms, $F$ belongs to a class defined by a weak form of sub-multiplicativity, and the $Q_{j}$ are arbitrary binary forms. A specific feature of the results is that the bounds are uniform within the $F$-class and that, as in a recent version given by Henriot, the dependency with respect to the coefficients of the $Q_{j}$ is made explicit. These estimates play a crucial rôle in the proof, published separately by the authors, of Manin's conjecture for Châtelet surfaces.
\end{abstract}

Keywords: Multiplicative functions, sub-multiplicative functions, short sums of arithmetic functions, binary forms, Hooley's Delta-function, divisors, count of lattice points over algebraic varieties.

Résumé. Généralisant des résultats classiques de Nair et Tenenbaum, nous fournissons des majorations générales et optimales pour des sommes du type

$$
\sum_{\substack{u<m \leqslant u+v \\ x<n \leqslant x+y}} F\left(Q_{1}(m, n), \ldots, Q_{k}(m, n)\right)
$$

où les paramètres $x, y, u, v$ ont des logarithmes comparables, $F$ décrit une classe de fonctions définie par une condition de sous-multiplicativité faible, et les $Q_{j}$ sont des formes binaires arbitraires. Ces résultats sont caractérisés par leur uniformité dans la classe des fonctions $F$ et, à l'instar d'une version récente donnée par Henriot, une dépendance explicite en fonction des coefficients des $Q_{j}$. Ces estimations jouent un rôle crucial dans la preuve, publiée séparément par les auteurs, de la conjecture de Manin pour les surfaces de Châtelet.

Mots-clefs : Fonctions multiplicatives, fonctions sous-multiplicatives, sommes courtes de fonctions arithmétiques, formes binaires, fonction Delta de Hooley, diviseurs, comptage de points entiers sur les variétés algébriques.

\section{Introduction}

L'étude des sommes courtes

$$
\sum_{x<n \leqslant x+y} f(n) \quad\left(x \geqslant 1, x^{\varepsilon}<y \leqslant x\right),
$$

où $f$ est une fonction multiplicative positive ou nulle et $\varepsilon>0$ désigne un nombre réel fixé, est cruciale dans beaucoup de problèmes de théorie analytique des nombres.

Le résultat de base de la théorie est celui de Shiu [20]. Cependant, au fil du développement de la théorie, les applications ont graduellement nécessité des résultats plus généraux. Nair [16] a obtenu des estimations valables pour des sommants de la forme $f(|Q(n)|)$ où $Q$ est un polynôme à coefficients entiers, et Nair \& Tenenbaum [17] ont traité le cas de fonctions de plusieurs variables d'arguments polynomiaux; de plus, leur méthode permet d'élargir le champ des fonctions $f$ bien au-delà de la sous-multiplicativité. Daniel, dans un manuscrit non publié datant du début des années 2000, puis, très récemment, 
Henriot [12] ont ensuite précisé la dépendance des constantes implicites entrant en jeu dans ces majorations en fonction du discriminant des polynômes considérés.

Ayant en vue une nouvelle application, décrite plus loin, relative à la preuve de la conjecture de Manin pour certaines surfaces de Châtelet, nous nous proposons ici de généraliser aux sommes de fonctions arithmétiques prenant pour valeurs des formes binaires, et avec la précision fournie dans [12], les estimations de [17].

Nos résultats, qui étendent directement ceux de [1], restreints aux fonctions sousmultiplicatives, sont applicables à une large classe de fonctions arithmétiques, intégrant, par exemple la fonction $\Delta$ de Hooley, qui entre dans le champ des hypothèses considérées dans [17]. Une telle généralité se révèle cruciale pour l'applications décrite plus haut.

Nous renvoyons à [12] pour une présentation plus détaillée de la bibliographie.

Un certain nombre de notations sont nécessaires pour énoncer nos résultats.

Pour $k \in \mathbb{N}^{*}, A \geqslant 1, B \geqslant 1, \varepsilon>0$, nous désignons $\mathcal{M}_{k}(A, B, \varepsilon)$ l'ensemble des fonctions arithmétiques $F$, positives ou nulles, de $k$ variables satisfaisant la condition

$$
F\left(a_{1} b_{1}, \ldots, a_{k} b_{k}\right) \leqslant \min \left\{A^{\Omega\left(a_{1} \cdots a_{k}\right)}, B\left(a_{1} \cdots a_{k}\right)^{\varepsilon}\right\} F\left(b_{1}, \ldots, b_{k}\right)
$$

pour tous $\boldsymbol{a}, \boldsymbol{b} \in \mathbb{N}^{* k}$ tels que $\left(a_{1} \cdots a_{k}, b_{1} \cdots b_{k}\right)=1$. Lorsque $F \neq 0$, nous posons

$$
G(\boldsymbol{a}):=\max _{\substack{b_{1}, \ldots, b_{k} \in \mathbb{N}^{*} \\\left(a_{1} \cdots a_{k}, b_{1} \cdots b_{k}\right)=1 \\ F\left(b_{1}, \ldots, b_{k}\right) \neq 0}} \frac{F\left(a_{1} b_{1}, \ldots, a_{k} b_{k}\right)}{F\left(b_{1}, \ldots, b_{k}\right)} \quad\left(\boldsymbol{a}=\left(a_{j}\right)_{1 \leqslant j \leqslant k} \in \mathbb{N}^{* k}\right) .
$$

Étant donnée une famille $Q_{1}, \ldots, Q_{k} \in \mathbb{Z}[X, Y]$ de $k$ formes binaires, nous posons

$$
Q=\prod_{1 \leqslant j \leqslant k} Q_{j}=\prod_{1 \leqslant h \leqslant r} R_{h}^{\gamma_{h}} \in \mathbb{Z}[X, Y], \quad g:=\operatorname{deg} Q,
$$

où les $R_{h}$ sont irréductibles dans $\mathbb{Z}[X, Y]$. Nous pouvons alors écrire canoniquement

$$
Q_{j}=\prod_{1 \leqslant h \leqslant r} R_{h}^{\gamma_{j h}} \quad(1 \leqslant j \leqslant k)
$$

où $\gamma_{j h} \geqslant 0$ pour tous $j, h$, de sorte que

$$
\gamma_{h}=\sum_{1 \leqslant j \leqslant k} \gamma_{j h} \quad(1 \leqslant h \leqslant r), \quad \sum_{1 \leqslant h \leqslant r} \gamma_{h}=g .
$$

Dans toute la suite nous nous plaçons dans le cas où la forme $Q$ est primitive, ce qui implique qu'il en va de même pour tous les $Q_{j}$.

Employant la notation

$$
\mathcal{D}(T):=\operatorname{disc}(T)
$$

pour désigner le discriminant d'un polynôme à une ou deux variables, de sorte que $\mathcal{D}(T(X, Y))=\mathcal{D}(T(X, 1))=\mathcal{D}(T(1, Y))$, nous posons

$$
D:=\mathcal{D}(Q), \quad D^{*}=\mathcal{D}\left(\prod_{1 \leqslant h \leqslant r} R_{h}\right),
$$

et notons que $D^{*} \neq 0$ par construction.

Lorsque $T \in \mathbb{Z}[X], s \in \mathbb{N}^{*}$, nous désignons par $\varrho_{T}(s)$ le nombre de racines de $T$ dans $\mathbb{Z} / s \mathbb{Z}$. Lorsque $T$ est une forme binaire de $\mathbb{Z}[X, Y]$, nous posons

$$
\varrho_{T}^{-}(s):=\sum_{\substack{1 \leqslant \xi \leqslant s \\ T(\xi, 1) \equiv 0(\bmod s)}} 1, \quad \varrho_{T}^{+}(s):=\sum_{\substack{1 \leqslant \xi, \eta \leqslant s \\ T(\xi, \eta) \equiv 0(\bmod s)}} 1 \quad(s \geqslant 1) .
$$


Désignons par $\kappa(s):=\prod_{p \mid s} p$ le noyau sans facteur carré d'un entier générique $s$ et posons

$$
\mathcal{K}(s):=\left[s_{1} \kappa\left(s_{1}\right), \ldots, s_{r} \kappa\left(s_{r}\right)\right] \quad\left(s=\left(s_{1}, \ldots, s_{r}\right) \in \mathbb{N}^{* r}\right) .
$$

Nous définissons alors les fonctions arithmétiques de $r$ variables

$$
\begin{aligned}
& \varrho_{\boldsymbol{R}}^{+}(s):=\sum_{\substack{1 \leqslant \xi, \eta \leqslant s_{1} \cdots s_{r} \\
R_{h}(\xi, \eta) \equiv 0\left(\bmod s_{h}\right)(1 \leqslant h \leqslant r)}} 1, \\
& \varrho_{\boldsymbol{R}}^{\#}(\boldsymbol{s}):=\sum_{\substack{1 \leqslant \xi, \eta \leqslant \mathcal{K}(s) \\
s_{h} \| R_{h}(\xi, \eta)(1 \leqslant h \leqslant r)}} 1, \quad\left(s=\left(s_{1}, \ldots, s_{r}\right) \in \mathbb{N}^{* r}\right)
\end{aligned}
$$

où le symbole $a \| b$ signifie que l'on a simultanément $a \mid b$ et $(a, b / a)=1$.

Étant donné $\boldsymbol{s}=\left(s_{1}, \ldots, s_{r}\right) \in \mathbb{N}^{* r}$, nous posons

$$
s_{j}^{\prime}:=\prod_{1 \leqslant h \leqslant r} s_{h}^{\gamma_{j h}}(1 \leqslant j \leqslant k), \quad s^{\prime \prime}:=\prod_{1 \leqslant j \leqslant k} s_{j}^{\prime}=\prod_{1 \leqslant h \leqslant r} s_{h}^{\gamma_{h}},
$$

de sorte que si, étant donné $(m, n) \in \mathbb{N}^{* 2}$, nous posons $s_{h}=R_{h}(m, n)(1 \leqslant h \leqslant r)$, alors $s_{j}^{\prime}=Q_{j}(m, n)$ pour tout $j \in[1, k]$ et $s^{\prime \prime}=Q(m, n)$. Avec ces notations, nous pouvons associer à toute fonction $F$ de $\mathcal{M}_{k}(A, B, \varepsilon)$ la fonction $\widehat{F}$ de $\mathcal{M}_{r}\left(A^{g}, B, g \varepsilon\right)$ définie par

$$
\widehat{F}\left(s_{1}, \ldots, s_{r}\right)=F\left(s_{1}^{\prime}, \ldots, s_{k}^{\prime}\right) .
$$

Nous désignons alors par $\widehat{G}$ la fonction associée à $\widehat{F}$ par $(1 \cdot 2)$ avec $k=r$ et notons $\widehat{G}_{h}: \mathbb{N}^{*} \rightarrow \mathbb{R}^{+}$la composée de $\widehat{G}$ et de la fonction $h$-ième coordonnée.

L'objet principal de ce travail consiste donc à estimer la quantité

$$
S:=\sum_{\substack{u<m \leqslant u+v \\ x<n \leqslant x+y}} F\left(Q_{1}(m, n), \ldots, Q_{k}(m, n)\right)=\sum_{\substack{u<m \leqslant u+v \\ x<n \leqslant x+y}} \widehat{F}\left(R_{1}(m, n), \ldots, R_{r}(m, n)\right) .
$$

Nous obtenons le résultat suivant, qui est le pendant bidimensionnel de celui de [12]. Nous notons $\|Q\|$ le maximum des valeurs absolues des coefficients d'une forme $Q$.

Théorème 1.1. Soit $k \in \mathbb{N}^{*}$, et $\left\{Q_{j}\right\}_{j=1}^{k} \in \mathbb{Z}[X, Y]^{k}$ une famille de polynômes homogènes primitifs. Définissons $Q \in \mathbb{Z}[X, Y],\left\{R_{h}\right\}_{h=1}^{r} \in \mathbb{Z}[X, Y]^{r}, g \in \mathbb{N}$ par (1·3), $\varrho_{Q}^{+}$par (1.4), et $\varrho_{R}^{\#}$ par (1·6). Pour tous

$$
\alpha \in] 0,1], \quad \delta \in] 0,1\left[, \quad A \geqslant 1, \quad B \geqslant 1, \quad 0<\varepsilon \leqslant \alpha \delta / 50 g^{2}(\delta g+1),\right.
$$

et uniformément sous les conditions

$$
F \in \mathcal{M}_{k}(A, B, \varepsilon), \quad \min (u, x) \geqslant c_{0} \max \{u, x,\|Q\|\}^{\delta}, \quad u^{\alpha} \leqslant v \leqslant u, \quad x^{\alpha} \leqslant y \leqslant x
$$
nous avons

$$
\sum_{\substack{u<m \leqslant u+v \\ x<n \leqslant x+y}} F\left(\left|Q_{1}(m, n)\right|, \ldots,\left|Q_{k}(m, n)\right|\right) \ll v y E_{\boldsymbol{R}}(u+x) \prod_{g<p \leqslant x}\left(1-\frac{\varrho_{Q}^{+}(p)}{p^{2}}\right)
$$


où $c_{0}$ et la constante implicite dépendent au plus de $g, \alpha, \delta, A, B$ et où l'on a posé

$$
E_{\boldsymbol{R}}(v):=\sum_{\substack{s \in \mathbb{N}^{* r} \\ s_{1} \cdots s_{r} \leqslant v}} \widehat{F}(s) \frac{\varrho_{\boldsymbol{R}}^{\#}(s)}{\mathcal{K}(s)^{2}} \quad(v \geqslant 1)
$$

Sous les mêmes hypothèses, nous avons

$$
\sum_{\substack{m \leqslant u \\ n \leqslant x}} F\left(\left|Q_{1}(m, n)\right|, \ldots,\left|Q_{k}(m, n)\right|\right) \ll u x E_{\boldsymbol{R}}(u+x) \prod_{g<p \leqslant x}\left(1-\frac{\varrho_{Q}^{+}(p)}{p^{2}}\right) .
$$

Comme dans [12], nous pouvons donner diverses formulations simplifiées pour la majoration.

Proposition 1.2. Dans les hypothèses du Théorème 1.1, nous avons

$$
E_{\boldsymbol{R}}(v) \ll \mathcal{H}\left(D^{*}\right) \sum_{\substack{s \in \mathbb{N}^{* r} \\ s_{1} \cdots s_{r} \leqslant v \\\left(s_{h}, D^{*}\right)=1(1 \leqslant h \leqslant r) \\\left(s_{i}, s_{j}\right)=1(1 \leqslant i<j \leqslant r)}} \widehat{F}(s) \prod_{1 \leqslant h \leqslant r} \frac{\varrho_{R_{h}}^{+}\left(s_{h}\right)}{s_{h}^{2}} \quad(v \geqslant 1)
$$

avec

$$
\mathcal{H}\left(D^{*}\right):=\prod_{p \mid D^{*}}\left(1+\sum_{\substack{\boldsymbol{\nu} \in \mathbb{N}^{r} \backslash\{\mathbf{0}\} \\ \nu_{h} \leqslant \operatorname{deg}\left(R_{h}\right)}} \widehat{G}\left(p^{\nu_{1}}, \ldots, p^{\nu_{r}}\right) \frac{\varrho_{\boldsymbol{R}}^{\#}\left(p^{\nu_{1}}, \ldots, p^{\nu_{r}}\right)}{p^{2 \max _{h} \nu_{h}+2}}\right),
$$

où la constante implicite dépend des mêmes paramètres qu'au Théorème 1.1. En particulier, on a

$$
\mathcal{H}\left(D^{*}\right) \leqslant \prod_{p \mid D^{*}}\left(1+\frac{1}{p}\right)^{\mathrm{e}^{2 g} A^{g}}
$$

L'hypothèse (1.1) permet également de majorer le second facteur de (1.12) par un produit eulérien.

Proposition 1.3. Dans les hypothèses du Théorème 1.1, nous avons

$$
E_{\boldsymbol{R}}(v) \ll \mathcal{H}\left(D^{*}\right) \prod_{1 \leqslant h \leqslant r} \prod_{\substack{g<p \leqslant v \\ p \nmid D^{*}}}\left(1+\widehat{G}_{h}(p) \frac{\varrho_{R_{h}}^{+}(p)}{p^{2}}\right) \quad(v \geqslant 1)
$$

où la constante implicite dépend des mêmes paramètres qu'au Théorème 1.1.

Nous pouvons exploiter la flexibilité des hypothèses du Théorème 1.1 pour traiter le cas d'arguments premiers. Dans l'énoncé suivant, les lettres $p$ et $q$ désignent des nombres premiers. 
Corollaire 1.4. Soient $k \in \mathbb{N}^{*}$, et $\left\{Q_{j}\right\}_{j=1}^{k} \in \mathbb{Z}[X, Y]^{k}$ une famille de polynômes homogènes primitifs. Conservons les notations du Théorème 1.1, supposons (1.7) et (1.8) vérifiées et, de plus, $Q(1,0) Q(0,1) \neq 0$ et $0<\varepsilon \leqslant \alpha \delta / 50(g+2)^{2}(\delta g+2 \delta+1)$. Nous avons alors

$$
\begin{aligned}
& \sum_{\substack{u<p \leqslant u+v \\
x<q \leqslant x+y}} F\left(\left|Q_{1}(p, q)\right|, \ldots,\left|Q_{k}(p, q)\right|\right) \\
& \qquad \frac{Q(1,0) Q(0,1) v y E_{\boldsymbol{R}}(u+x)}{\varphi(Q(1,0)) \varphi(Q(0,1))\{\log (u+x)\}^{2}} \prod_{g<p \leqslant x}\left(1-\frac{\varrho_{Q}^{+}(p)}{p^{2}}\right),
\end{aligned}
$$

où $c_{0}$ et la constante implicite dépendent au plus de $g, \alpha, \delta, A, B$.

Sous les mêmes hypothèses, nous avons

$$
\begin{aligned}
\sum_{\substack{p \leqslant u \\
q \leqslant x}} F\left(\left|Q_{1}(p, q)\right|\right. & \left.\ldots,\left|Q_{k}(p, q)\right|\right) \\
& \ll \frac{Q(1,0) Q(0,1) u x E_{\boldsymbol{R}}(u+x)}{\varphi(Q(1,0)) \varphi(Q(0,1))\{\log (u+x)\}^{2}} \prod_{g<p \leqslant x}\left(1-\frac{\varrho_{Q}^{+}(p)}{p^{2}}\right) .
\end{aligned}
$$

Nous sommes à présent en mesure de décrire l'application principale envisagée, relative à la preuve de la conjecture de Manin pour certaines variétés algébriques.

Introduisons en premier lieu la classe des fonctions arithmétiques auxquelles nous appliquerons le Théorème 1.1. Étant donnée une fonction arithmétique auxiliaire $f$, nous posons

$$
\begin{gathered}
\Delta(n, f ; u, v):=\sum_{\substack{d \mid n \\
\mathrm{e}^{u}<d \leqslant \mathrm{e}^{u+v}}} f(d), \quad\left(n \in \mathbb{N}^{*}, u \in \mathbb{R}, v>0\right), \\
\Delta(n, f):=\sup _{u \in \mathbb{R}, 0 \leqslant v \leqslant 1}|\Delta(n, f ; u, v)| \quad\left(n \in \mathbb{N}^{*}\right) .
\end{gathered}
$$

Ainsi, les fonction $\Delta(n, f)$ sont des généralisations de la fonction $\Delta$ de Hooley, correspondant au cas $f=\mathbf{1}$ - cf. notamment [13], [11], [14] et les références indiquées dans ces travaux.

Les fonctions arithmétiques du type $F(n):=\Delta(n, f)$ ne satisfont pas les hypothèses de [1] mais, lorsque, par exemple, $f$ est à valeurs dans le disque unité, nous avons

$$
\Delta(a b, f) \leqslant \tau(a) \Delta(b, f) \quad((a, b)=1)
$$

de sorte que $F \in \mathcal{M}_{1}(2, B, \varepsilon)$ pour tout $\varepsilon>0$ et $B=B_{\varepsilon}$ convenable. Ces fonctions $F$ relèvent donc des résultats présentés plus haut.

La conjecture de Manin est une hypothèse générale concernant la répartition des points rationnels sur une large classe de variétés projectives $V$ pour lesquelles l'ensemble des points rationnels $V(\mathbb{Q})$ est dense dans $V$. Elle propose, pour tout ouvert convenablement choisi $U \subset V$, une formule asymptotique pour le nombre $N(B)$ des points de $U \cap V(\mathbb{Q})$ dont la hauteur n'excède pas un paramètre $B$. Le terme principal attendu pour $N(B)$ est alors de la forme $C B(\log B)^{r-1}$, où $r$ et $C$ possèdent des interprétations géométriques précises.

Une surface de Châtelet $V$ sur $\mathbb{Q}$ est un modèle propre et lisse de la surface affine

$$
y^{2}+z^{2}=P(x, 1)
$$


où $P \in \mathbb{Z}[X, Y]$ est une forme binaire de degré 4 de discriminant non nul. Le choix de la hauteur étant effectué (voir [7]), le cardinal à estimer dans le contexte de la conjecture de Manin $^{(1)}$ est

$$
N(B):=\frac{1}{2} \sum_{\substack{(y, z, t, m, n) \in \mathbb{Z}^{5}, t>0 \\ \max \left\{m^{2}, n^{2}\right\} t \leqslant B \\ y^{2}+z^{2}=t^{2} P(m, n) \\(m, n)=(y, z, t)=1}} 1 .
$$

Le cas où $P$ est scindé a été traité dans [5], et celui où $P$ possède un facteur cubique irréductible dans [4]. La situation où $P$ est le produit de deux formes linéaires et d'un facteur quadratique relève des méthodes de [5] et [4] : une adaptation standard utilisant le formalisme développé dans [5] permet d'obtenir le résultat souhaité. Nos résultats permettent de disposer des deux cas résiduels, correspondant à la situation où $P$ est irréductible sur $\mathbb{Q}[i]$ ou est le produit de deux formes $P_{1}$ et $P_{2}$ de degré 2 irréductibles $\operatorname{sur} \mathbb{Q}[i]$.

L'évaluation de $N(B)$ peut être réduite à l'estimation asymptotique des sommes de la forme

$$
\sum_{(m, n) \in \mathbb{Z}^{2} \cap \mathcal{R}} r(P(m, n))
$$

où $r(s):=\operatorname{card}\left\{(a, b) \in \mathbb{Z}^{2}: s=a^{2}+b^{2}\right\}$ et $\mathcal{R}$ est une région de $\mathbb{R}^{2}$. Lorsque $P$ est scindé, cette estimation a été obtenue dans [2]; le cas où $P$ possède un facteur cubique irréductible a été traité dans [4].

Pour aborder les autres cas, il est nécessaire de recourir à une majoration en moyenne de $\Delta(P(m, n), \chi)^{2}$ lorsque $P$ est irréductible - resp. de $r\left(P_{1}(m, n)\right) \Delta\left(P_{2}(m, n), \chi\right)^{2}$ lorsque $P$ est produit de deux formes irréductibles $P_{1}$ et $P_{2}$ de degré 2 - fournissant l'ordre de grandeur correct. Ainsi, l'application envisagée - nécessitant d'une part de pouvoir choisir $k \geqslant 2$ et, d'autre part, de prendre en compte des fonctions significativement plus générales que les fonctions sous-multiplicatives - a-t-elle largement motivé le cadre de cette étude.

Présentons succinctement la démarche développée dans [7] lorsque $P$ est irréductible de degré 4 sur $\mathbb{Q}[i]$. Le point de départ consiste à utiliser la formule classique

$$
r(s)=4 \sum_{d \mid s} \chi(d)
$$

où $\chi$ est l'unique caractère de Dirichlet non principal de module 4. Des résultats de géométrie des nombres permettant de déterminer un bon niveau de répartition, relativement à la variable $d$, pour la quantité

$$
\sum_{\substack{(m, n) \in \mathbb{Z}^{2} \cap \mathcal{R} \\ d \mid P(m, n)}} 1
$$

il est possible, lorsque, disons, $\mathcal{R}$ est une région telle que $\sup _{(m, n) \in \mathcal{R}} P(m, n) \leqslant X^{4}$ et $V$ est de la forme $V=X^{2} /(\log X)^{c}$, de traiter classiquement le cas où $d \notin\left[V, X^{4} / V\right]$.

1. Des détails supplémentaires relatifs à la conjecture de Manin dans le cas particulier des surfaces de Châtelet peuvent être trouvés dans [5]. 
Le point délicat consiste à estimer la contribution complémentaire, soit $S_{0}$. Posant

$$
\mathcal{B}:=\left\{(m, n) \in \mathbb{Z}^{2} \cap \mathcal{R}: \exists d \in\left[V, X^{4} / V\right]: d \mid P(m, n)\right\},
$$

nous obtenons par l'inégalité de Cauchy-Schwarz

$$
S_{0} \ll|\mathcal{B}|^{1 / 2} \log \left(\mathrm{e} X^{4} / V^{2}\right) M_{2}^{1 / 2}
$$

où l'on a posé

$$
M_{2}:=\sum_{(m, n) \in \mathbb{Z}^{2} \cap \mathcal{R}} \Delta(P(m, n), \chi)^{2} .
$$

Une estimation satisfaisante de $M_{2}$ peut être déduite des majorations pour les moments de la fonction $\Delta(\cdot, \chi)$ obtenues dans [6] et des résultats établis dans le présent travail. Il est à noter que, pour l'utilisation de nos résultats en vue de majorer $|\mathcal{B}|$, un choix explicite du paramètre $\varepsilon>0$ apparaissant dans l'énoncé du Théorème 1.1 est nécessaire : en l'occurrence, il s'agit de garantir que certaines fonctions de la forme $y^{\Omega_{\xi}(s)}$ avec

$$
\Omega_{\xi}(s):=\sum_{\substack{p^{\nu} \| s \\ p>\xi}} \nu
$$

satisfont aux hypothèses du Théorème 1.1 pour $\xi$ suffisamment grand. On voit ainsi que la forme particulière des conditions $(1 \cdot 1)$, contrastant, par exemple, avec les hypothèses classiques de [20], joue ici un rôle essentiel.

Nous obtenons dans [7] le résultat suivant.

Théorème 1.5. Soit $P$ une forme binaire de degré 4 de $\mathbb{Z}[X, Y]$, irréductible sur $\mathbb{Q}[i]$ ou produit de deux formes binaires de degré 2 irréductibles sur $\mathbb{Q}[i]$ non proportionnelles. Pour une constante convenable $C=C_{P}>0$, nous avons, lorsque $B$ tend vers l'infini,

$$
N(B)=B \log B\left\{C_{P}+O\left((\log B)^{-1 / 100}\right)\right\},
$$

où $C_{P}$ est la constante conjecturée par Peyre dans [19]. ${ }^{(2)}$

\section{Estimations relatives au nombre de solutions modulo $s$ d'une équation polynomiale}

Ce paragraphe est consacré au rappel de quelques résultats essentiellement classiques.

Lemme 2.1. Soit $T \in \mathbb{Z}[X]$ un polynôme primitif de degré $d$ et de discriminant $\mathcal{D}(T)$ non nul. Si $p^{\mu} \| \mathcal{D}(T)$, nous avons

$$
\varrho_{T}\left(p^{\nu}\right) \leqslant d p^{\min \{\nu-1,\lfloor(1-1 / d) \nu\rfloor, 2 \mu\}} .
$$

Démonstration. L'inégalité $\varrho_{T}\left(p^{\nu}\right) \leqslant d p^{\lfloor(1-1 / d) \nu\rfloor}$ est due à Stewart [21], les autres peuvent être trouvées dans le livre de Nagell [15].

Étant donné $T \in \mathbb{Z}[X, Y]$, nous introduisons, lorsque $s \in \mathbb{N}$, les ensembles

$$
\Lambda(s):=\left\{(m, n) \in \mathbb{Z}^{2}: s \mid T(m, n)\right\}, \quad \Lambda^{*}(s):=\{(m, n) \in \Lambda(s):(m, n, s)=1\},
$$

de sorte que l'on a $\varrho_{T}^{+}(s):=\mid \Lambda(s) \cap\left[0, s\left[^{2} \mid\right.\right.$ avec la notation introduite en (1.4). Nous posons également

$$
\varrho_{T}^{*}(s):=\mid \Lambda^{*}(s) \cap\left[0, s\left[^{2} \mid\right.\right.
$$

2. Voir [7] pour plus d'information sur la constante de Peyre. 
Lemme 2.2. Soit $T \in \mathbb{Z}[X, Y]$ une forme binaire primitive de degré $d$ et de discriminant $\mathcal{D}(T)$ non nul. Pour tout nombre premier $p$, nous avons

$$
\begin{aligned}
\varrho_{T}^{+}\left(p^{\nu}\right) & =\sum_{0 \leqslant k \leqslant\lceil\nu / d\rceil} p^{2 \min \{\nu, d k\}-2 k} \varrho_{T}^{*}\left(p^{\max \{\nu-d k, 0\}}\right) \\
& \leqslant(2 d+1) p^{\min \{2 \nu-1,\lfloor(2-1 / d) \nu\rfloor\}}
\end{aligned}
$$

Soit $c_{0}:=T(1,0)$. Lorsque $p \nmid c_{0} \mathcal{D}(T)$ et $\nu \geqslant 1$, nous avons

$$
\varrho_{T}^{+}\left(p^{\nu}\right)= \begin{cases}p^{\nu} \varrho_{T}^{-}(p) \frac{p^{(d-2)\lceil\nu / d\rceil}-1}{p^{d-2}-1}+p^{2(\nu-\lceil\nu / d\rceil)} & \text { si } d \geqslant 3 ; \\ p^{\nu} \varrho_{T}^{-}(p)\lceil\nu / 2\rceil(1-1 / p)+p^{2(\nu-\lceil\nu / 2\rceil)} & \text { si } d=2 ; \\ p^{\nu} & \text { si } d=1,\end{cases}
$$

de sorte que $\varrho_{T}^{+}(p)=(p-1) \varrho_{T}^{-}(p)+1$ lorsque $p \nmid c_{0} \mathcal{D}(T)$.

Lorsque $p \nmid \mathcal{D}(T)$ et $\nu \geqslant 1$, nous avons

$$
\varrho_{T}^{+}\left(p^{\nu}\right) \leqslant \begin{cases}2 d p^{\nu} \frac{p^{(d-2)\lceil\nu / d\rceil}-1}{p^{d-2}-1}+p^{2(\nu-\lceil\nu / d\rceil)} & \text { si } d \geqslant 3 ; \\ 2 d p^{\nu}\lceil\nu / 2\rceil(1-1 / p)+p^{2(\nu-\lceil\nu / 2\rceil)} & \text { si } d=2 ; \\ p^{\nu} & \text { si } d=1 .\end{cases}
$$

Démonstration. L'égalité de $(2 \cdot 3)$ est obtenue en sommant sur les entiers $k$ tels que $p^{k} \|(m, n)$. Pour obtenir l'inégalité, nous observons que

$$
\varrho_{T}^{*}\left(p^{\nu}\right) \leqslant \varphi\left(p^{\nu}\right)\left(\varrho_{T(X, 1)}\left(p^{\nu}\right)+\varrho_{T(1, X)}\left(p^{\nu}\right)\right) \leqslant 2 d \varphi\left(p^{\nu}\right) p^{\min \{\nu-1,\lfloor(1-1 / d) \nu\rfloor\}},
$$

d'après $(2 \cdot 1)$, et nous reportons dans l'égalité précédente.

La formule $(2 \cdot 4)$ est déduite de $(2 \cdot 3)$ et de la relation

$$
\varrho_{T}^{*}\left(p^{\nu}\right)=\varphi\left(p^{\nu}\right) \varrho_{T}(p) \quad\left(p \nmid c_{0} \mathcal{D}(T)\right) .
$$

Enfin $(2 \cdot 5)$ découle de la majoration

$$
\varrho_{T}^{*}\left(p^{\nu}\right) \leqslant 2 d \varphi\left(p^{\nu}\right) \quad(p \nmid \mathcal{D}(T)) .
$$

\section{Démonstrations}

\subsection{Preuve du Théorème 1.1}

Le résultat suivant est classique

Lemme 3.1. Soit $Q \in \mathbb{Z}[X, Y]$ une forme binaire primitive dont une décomposition en facteurs irréductibles s'écrit $Q=R_{1}^{\gamma_{1}} \cdots R_{r}^{\gamma_{r}}$. Alors, pour tout $n \in \mathbb{Z}^{*}$, la décomposition en facteurs irréductibles dans $\mathbb{Q}[X]$ de $Q(X, n)$ s'écrit

$$
Q(X, n)=\prod_{1 \leqslant h \leqslant r} R_{h}(X, n)^{\gamma_{h}}
$$

De plus, si $q_{j}(n)$ désigne le contenu de $R_{j}(X, n)$, le polynôme $R_{j}^{*}(X, n):=R_{j}(X, n) / q_{j}(n)$ est irréductible dans $\mathbb{Z}[X]$.

Soit $Q(X, Y):=\sum_{0 \leqslant j \leqslant d} c_{j} X^{d-j} Y^{j}$, de sorte que

$$
q(n)=\operatorname{pgcd}\left(c_{0}, c_{1} n, \ldots, c_{d-1} n^{d-1}, c_{d} n^{d}\right)=\prod_{1 \leqslant h \leqslant r} q_{h}(n)^{\gamma_{h}}
$$

d'après le lemme de Gauss. Nous posons

$$
Q^{*}(X, n)=Q(X, n) / q(n),
$$

qui est donc, pour chaque valeur du paramètre $n \in \mathbb{Z}^{*}$, un polynôme primitif de $X$. 
Nous rappelons ci-dessous le lemme 6 de [12] concernant des polynômes à une variable. Notons

$$
\varepsilon_{1}:=\frac{3}{25} \alpha, \quad \varepsilon_{2}:=\alpha /(50 g) .
$$

Lemme 3.2 [12]. Soit $T \in \mathbb{Z}[X]$ un polynôme de degré $g$, dont une décomposition en facteurs irréductibles de $\mathbb{Z}[X]$ est $T=\prod_{1 \leqslant h \leqslant r} R_{h}$. Pour $\boldsymbol{a} \in \mathbb{N}^{* r}, a_{1} \cdots a_{r} \leqslant x^{\varepsilon_{1}}, z \leqslant x^{\varepsilon_{2}}$, et $x^{\alpha} \leqslant y \leqslant x$, nous avons

$$
\sum_{\substack{x<n \leqslant x+y \\ a_{h} \| R_{h}(n)(1 \leqslant h \leqslant r) \\(p \mid T(n), g<p \leqslant z) \Rightarrow p \mid a_{1} \cdots a_{r}}} 1 \ll y \frac{\varrho_{\boldsymbol{R}}^{\#}(\boldsymbol{a})}{\mathcal{K}(\boldsymbol{a})} \prod_{\substack{g<p \leqslant z \\ p \nmid a_{1} \cdots a_{r}}}\left(1-\frac{\varrho_{T}(p)}{p}\right)
$$

où la constante implicite dépend au plus de $\alpha$ et $g$.

Il est à noter que

$$
\frac{\varrho_{\boldsymbol{R}}^{\#}(\boldsymbol{a})}{\mathcal{K}(\boldsymbol{a})}=\operatorname{dens}\left\{n \in \mathbb{Z}: a_{h} \| R_{h}(n)(1 \leqslant h \leqslant r)\right\} .
$$

La condition $p>g$ dans (3.1) permet d'éviter les éventuels diviseurs premiers fixes des $R_{h}$.

Nous observons par ailleurs que la même démonstration permet de remplacer, dans les conditions de sommation du membre de gauche de $(3 \cdot 1)$, la relation $a_{h} \| R_{h}(n)$ par

$$
a_{h}=a_{h}^{\prime} a_{h}^{\prime \prime}, \quad a_{h}^{\prime} \mid R_{h}(n), \quad\left(a_{h}, R_{h}(n) / a_{h}^{\prime}\right)=1 \quad(1 \leqslant h \leqslant r) .
$$

Nous considérons maintenant des polynômes à deux variables. L'analogue du Lemme 3.2 s'énonce de la manière suivante.

Lemme 3.3. Soit $Q \in \mathbb{Z}[X, Y]$ une forme binaire satisfaisant aux hypothèses du Théorème 1.1. Pour $\boldsymbol{a} \in \mathbb{N}^{* r}, u \geqslant 1, x \geqslant 1, w:=\min (u, x), a_{1} \cdots a_{r} \leqslant w^{\varepsilon_{1}}, z \leqslant w^{\varepsilon_{2}}$, $u^{\alpha} \leqslant v \leqslant u, x^{\alpha} \leqslant y \leqslant x$, nous avons

$$
\sum_{\substack{u<m \leqslant u+v \\ x<n \leqslant x+y \\ a_{h} \| R_{h}(m, n)(1 \leqslant h \leqslant r)}} 1 \ll v y \frac{\varrho_{\boldsymbol{R}}^{\#}(\boldsymbol{a})}{\mathcal{K}(\boldsymbol{a})^{2}} \prod_{\substack{g<p \leqslant z \\ p \nmid a_{1} \cdots a_{r}}}\left(1-\frac{\varrho_{Q}^{+}(p)}{p}\right)
$$

Supposant momentanément ce résultat établi, nous sommes en mesure de prouver le Théorème 1.1. Il suffit, en effet, de suivre, mutatis mutandis, la preuve du théorème 5 de [12] en remplaçant l'utilisation du Lemme 3.2 par celle du Lemme 3.3. Nous n'indiquons pas plus de détails.

\subsection{Démonstration du Lemme 3.3}

Rappelons que l'ensemble des diviseurs premiers fixes d'un polynôme primitif de degré $\leqslant g$ est toujours inclus dans $[2, g]$. Avec les notations introduites au Lemme 3.1, la condition $a_{h} \| R_{h}(m, n)$ s'écrit $a_{h}=a_{h}^{\prime} a_{h}^{\prime \prime}$ et

$$
a_{h}^{\prime} \mid R_{h}^{*}(m, n), \quad\left(a_{h}, R_{h}^{*}(m, n) / a_{h}^{\prime}\right)=1, \quad\left(a_{h}, q_{h}(n) / a_{h}^{\prime \prime}\right)=1 .
$$

Lorsque $\ell=(\mathcal{K}(\boldsymbol{a}), n)$ est fixé, les conditions $(3 \cdot 3)$ sont équivalentes à

$$
a_{h}^{\prime} \mid R_{h}^{*}(m, \ell), \quad\left(a_{h}, R_{h}^{*}(m, \ell) / a_{h}^{\prime}\right)=1, \quad\left(a_{h}, q_{h}(\ell) / a_{h}^{\prime \prime}\right)=1 .
$$


De plus, la condition $(p \mid Q(m, n), g<p \leqslant z) \Rightarrow p \mid a_{1} \cdots a_{r}$ est équivalente à

$$
\left(p \mid\left(c_{0}, n\right) Q^{*}(m, n), g<p \leqslant z\right) \Rightarrow p \mid a_{1} \cdots a_{r} .
$$

Nous avons

$$
\begin{aligned}
\frac{\varrho_{\boldsymbol{R}}^{*}(\boldsymbol{a})}{\mathcal{K}(\boldsymbol{a})^{2}} & =\operatorname{dens}\left\{(m, n) \in \mathbb{Z}^{2}: a_{h} \| R_{h}(m, n) \quad(h \leqslant r)\right\} \\
& =\sum_{a_{h}^{\prime} a_{h}^{\prime \prime}=a_{h}} \sum_{\begin{array}{c}
\ell \mid \mathcal{K}(\boldsymbol{a}) \\
\left(a_{h}, q_{h}(\ell) / a_{h}^{\prime \prime}\right)=1
\end{array}} \beta(\ell) \operatorname{dens}\left\{n \in \mathbb{N}^{*}: \ell=(\mathcal{K}(\boldsymbol{a}), n)\right\}
\end{aligned}
$$

où l'on a posé

$$
\beta(\ell):=\operatorname{dens}\left\{m: a_{h}^{\prime} \mid R_{h}^{*}(m, \ell), \quad\left(a_{h}, R_{h}^{*}(m, \ell) / a_{h}^{\prime}\right)=1\right\} .
$$

Nous sommes maintenant en mesure d'appliquer la variante du Lemme 3.2 explicitée plus haut. Lorsque

$$
a_{h}=a_{h}^{\prime} a_{h}^{\prime \prime}, \quad \ell \mid \mathcal{K}(\boldsymbol{a}), \quad\left(a_{h}, q_{h}(\ell) / a_{h}^{\prime \prime}\right)=1, \quad \ell=(\mathcal{K}(\boldsymbol{a}), n),
$$

et que l'entier $n$ satisfait (3.5), nous obtenons

$$
\begin{gathered}
\sum_{\substack{u<m \leqslant u+v \\
a_{h}^{\prime} \mid R_{h}^{*}(m, \ell) \\
(1 \leqslant h \leqslant r)}} 1 \ll v \beta(m) \prod_{\substack{g<p \leqslant z \\
p \nmid a_{1} \cdots a_{r}}}\left(1-\frac{\varrho_{Q^{*}(\cdot, n)}(p)}{p}\right) . \\
\begin{array}{c}
\left(a_{h}, R_{h}^{*}(m, \ell) / a_{h}^{\prime}\right)=1(1 \leqslant h \leqslant r) \\
\left(p \mid Q^{*}(m, n), g<p \leqslant z\right) \Rightarrow p \mid a_{1} \cdots a_{r}
\end{array}
\end{gathered}
$$

Lorsque $p \nmid \mathcal{K}(\boldsymbol{a}), g<p \leqslant z$, nous avons nécessairement $p \nmid q(n)$. Posant $\delta_{p}\left(c_{0}\right):=$ $\mathbf{1}_{\mathbb{Z}}\left(c_{0} / p\right)$, il suit

$$
\frac{\varrho_{Q^{*}(\cdot, n)}(p)}{p}=\frac{\varrho_{Q}^{+}(p)}{p^{2}}-\frac{\delta_{p}\left(c_{0}\right)}{p},
$$

et donc

$$
\sum_{\substack{u<m \leqslant u+v \\ a_{h}^{\prime}\left|R_{h}^{*}(m, \ell)(1 \leqslant h \leqslant r) \\\left(a_{h}, R_{h}^{*}(m, \ell) / h_{h}^{\prime}\right)=1(1 \leqslant h \leqslant r) \\\left(p \mid Q^{*}(m, n), g<p \leqslant z\right) \Rightarrow p\right| a_{1} \cdots a_{r}}} 1 \ll v \beta(\ell) \prod_{\substack{g<p \leqslant z \\ p \nmid a_{1} \cdots a_{r}}}\left(1-\frac{\varrho_{Q}^{+}(p)}{p^{2}}\right) \prod_{\substack{g<p \leqslant z \\ p \nmid a_{1} \cdots a_{r}}}\left(1+\frac{\delta_{p}\left(c_{0}\right)}{p}\right) .
$$

Il reste à sommer sur $n$. Un argument de crible standard permet d'écrire, sous les hypothèses effectuées,

$$
\sum_{\substack{x<n \leqslant x+y \\(\mathcal{K}(\boldsymbol{a}), n)=\ell \\ g<p \leqslant z) \Rightarrow p \mid a_{1} \cdots a_{r}}} 1 \ll y \operatorname{dens}\left\{n \in \mathbb{N}^{*}:(\mathcal{K}(\boldsymbol{a}), n)=\ell\right\} \prod_{\substack{g<p \leqslant z \\ p \nmid a_{1} \cdots a_{r}}}\left(1-\frac{\delta_{p}\left(c_{0}\right)}{p}\right) .
$$

Cela fournit le résultat annoncé (1.9) après sommation sur $a_{h}^{\prime}, a_{h}^{\prime \prime}, \ell$ et recours à la formule $(3 \cdot 6)$.

L'estimation (1.11) peut être facilement déduite de (1.9) en scindant les intervalles $[1, u]$ et $[1, x]$ en intervalles dyadiques et en majorant trivialement la contribution du domaine $\max (u / m, x / n) \geqslant(u+x)^{(g \delta+1) \varepsilon}$. 


\section{3. Preuve de la Proposition 1.2}

Pour $1 \leqslant h \leqslant r$, écrivons $s_{h}=s_{h}^{\prime} s_{h}^{\prime \prime}$ avec $\left(s_{h}^{\prime}, D^{*}\right)=1$ et $s_{h}^{\prime \prime} \mid D^{* \infty}$. Notant $w:=u+x$, nous avons

$$
E_{\boldsymbol{R}}(w) \ll \mathcal{H} \mathcal{H}^{\prime} \quad \sum_{\substack{s_{1} \cdots s_{r} \leqslant w \\\left(s_{h}, D^{*}\right)=1(1 \leqslant h \leqslant r) \\\left(s_{i}, s_{j}\right)=1(i \neq j)}} \widehat{F}(s) \prod_{1 \leqslant h \leqslant r} \frac{\varrho_{R_{h}}^{+}\left(s_{h}\right)}{s_{h}^{2}}
$$

où l'on a posé $\mathcal{H}:=\prod_{p \mid D^{*}} \mathcal{H}_{p}, \mathcal{H}^{\prime}:=\prod_{p \nmid D^{*}} \mathcal{H}_{p}^{\prime}$, avec

$$
\mathcal{H}_{p}:=1+\sum_{\boldsymbol{\nu} \in \mathbb{N}^{r} \backslash\{\mathbf{0}\}} \widehat{G}\left(p^{\nu_{1}}, \ldots, p^{\nu_{r}}\right) \frac{\varrho_{\boldsymbol{R}}^{\#}\left(p^{\nu_{1}}, \ldots, p^{\nu_{r}}\right)}{p^{2 \max \nu_{h}+2}},
$$

et

$$
\mathcal{H}_{q}:=1+\sum_{\substack{\boldsymbol{\nu} \in \mathbb{N}^{r} \backslash\{0\} \\\left|\left\{h \in[1, r]: \nu_{h} \geqslant 1\right\}\right| \geqslant 2}} \widehat{G}\left(p^{\nu_{1}}, \ldots, p^{\nu_{r}}\right) \frac{\varrho_{\boldsymbol{R}}^{\#}\left(p^{\nu_{1}}, \ldots, p^{\nu_{r}}\right)}{p^{2 \max \nu_{h}+2}} .
$$

Notons $g_{h}:=\operatorname{deg}\left(R_{h}\right) \quad(1 \leqslant h \leqslant r)$. Dans un premier temps, nous majorons la contribution $\mathcal{H}_{p}^{\dagger}$ à $\mathcal{H}_{p} \mathcal{H}_{p}^{\prime}$ des multi-indices $\boldsymbol{\nu}$ tels que $\max _{h} \nu_{h} / g_{h}>1$. Si le maximum est atteint en $h=t$, nous pouvons écrire

$$
\frac{\varrho_{R}^{\#}\left(p^{\nu_{1}}, \ldots, p^{\nu_{r}}\right)}{p^{2 \max \nu_{h}+2}} \leqslant \frac{\varrho_{R_{t}}^{+}\left(p^{\nu_{t}}\right)}{p^{2 \nu_{t}}} \leqslant\left(2 g_{t}+1\right) p^{-\nu_{t} / g_{t}}
$$

et

$$
\widehat{G}\left(p^{\nu_{1}}, \ldots, p^{\nu_{r}}\right) \ll B p^{\varepsilon g^{2} \nu_{t} / g_{t}},
$$

puisque $\sum_{h} \nu_{h} \leqslant \sum_{h} g_{h} \nu_{t} / g_{t}=g \nu_{t} / g_{t}$. Il suit

$$
\mathcal{H}_{p}^{\dagger} \ll B \sum_{\nu_{h}>g_{h}} p^{\varepsilon g^{2} \nu_{h} / g_{h}} \frac{\left(\nu_{h} / g_{h}+1\right)^{r}}{p^{\nu_{h} / g_{h}}} \prod_{1 \leqslant h \leqslant r} g_{h} \ll_{g, B} \frac{1}{p^{1+1 / 2 g}}
$$

dès que $\varepsilon \leqslant 1 / 2 g^{3}$.

Ainsi $\prod_{p}\left(1+\mathcal{H}_{p}^{\dagger}\right)<\infty$, et nous pouvons négliger cette contribution dans le produit $\mathcal{H H}^{\prime}$.

Estimons la contribution $\mathcal{H}_{p}^{\ddagger}$ à $\mathcal{H}_{p}^{\prime}$ des multi-indices $\boldsymbol{\nu}$ tels que $\max _{h} \nu_{h} / g_{h} \leqslant 1$. Supposons que $\nu_{t}=\min \left\{\nu_{h}: \nu_{h} \geqslant 1\right\}$. Il existe alors, par hypothèse, un second indice, disons $s$, tel que $\nu_{s} \geqslant \nu_{t} \geqslant 1$.

Comme $p \nmid D^{*}$, les relations $p^{\nu_{t}}\left|R_{t}(m, n), p^{\nu_{s}}\right| R_{j}(m, n)$ et $\nu_{s} \geqslant \nu_{t} \geqslant 1$ impliquent $p^{\nu_{t}} \mid(m, n)$. D'où

$$
\frac{\varrho_{R}^{\#}\left(p^{\nu_{1}}, \ldots, p^{\nu_{r}}\right)}{p^{2 \max _{j} \nu_{j}+2}} \leqslant \frac{1}{p^{2 \nu_{t}}} .
$$

Comme $\sum_{h} \nu_{h} \leqslant \sum_{h} g_{h} \leqslant g$, nous avons en outre $\widehat{G}\left(p^{\nu_{1}}, \ldots, p^{\nu_{r}}\right) \ll B p^{\varepsilon g^{2}}$. Nous obtenons donc

$$
\mathcal{H}_{p}^{\ddagger} \ll g, B \frac{1}{p^{1+1 / 2 g_{h}}}+\frac{p^{\varepsilon g^{2}}}{p^{2}} .
$$

Ainsi, $\prod_{p}\left(1+\mathcal{H}_{p}^{\ddagger}\right)<\infty$, et nous obtenons $\mathcal{H}^{\prime} \ll 1$, d'où résulte la première assertion du Proposition 1.2.

Il reste à estimer $\mathcal{H}_{p}-1$ lorsque $p \mid D^{*}$. D'après le Lemme 2.2 , nous avons

$$
\mathcal{H}_{p}-1 \leqslant \frac{A^{g}}{p} \prod_{j}\left(g_{j}+1\right)\left(2 g_{j}+1\right) \leqslant \frac{\mathrm{e}^{2} A^{g}}{p},
$$

où nous avons utilisé les inégalités $(k+1)(2 k+1) \leqslant \mathrm{e}^{2 k}(k \geqslant 0)$ et $\sum_{h} g_{h} \leqslant g$. Cela permet d'en déduire (1·13) et achève ainsi la démonstration de la Proposition 1.2. 


\subsection{Démonstration de la Proposition 1.3}

La somme en $s$ apparaissant au membre de droite de (1.12) n'excède pas

$$
\prod_{\substack{g<p \leqslant x \\ p \nmid D^{*}}}\left(1+\sum_{\nu_{h} \geqslant 1} \widehat{G}_{h}\left(p^{\nu_{h}}\right) \frac{\varrho_{R_{h}}^{+}\left(p^{\nu_{h}}\right)}{p^{2 \nu_{h}}}\right) \ll \prod_{\substack{g<p \leqslant x \\ p \nmid D^{*}}}\left(1+\sum_{1 \leqslant \nu_{h} \leqslant g_{h}} \widehat{G}_{h}\left(p^{\nu_{h}}\right) \frac{\varrho_{R_{h}}^{+}\left(p^{\nu_{h}}\right)}{p^{2 \nu_{h}}}\right) .
$$

où la seconde estimation majoration a été obtenue par une manipulation analogue à celle de la démonstration du Proposition 1.2.

Puisque $\mathcal{D}\left(R_{h}\right) \mid D^{*}$, les majorations $(2.5)$ permettent d'établir que la contribution globale des $\nu_{h} \geqslant 2$ peut être englobée par la constante implicite. Nous omettons les détails de la vérification.

\subsection{Preuve du Corollaire 1.4}

Soit $\chi$ la fonction indicatrice de l'ensemble des entiers sans facteur premier dans l'intervalle $[2, \min \{u, x\}]$. Posant $Q_{k+1}(X, Y):=X, Q_{k+2}(X, Y):=Y$, nous appliquons le Théorème 1.1 à la fonction

$$
\left(s_{1}, \ldots, s_{k+2}\right) \mapsto F\left(s_{1}, \ldots, s_{k}\right) \chi\left(s_{k+1}\right) \chi\left(s_{k+2}\right)
$$

et à la famille de polynômes $\left\{Q_{j}\right\}_{j=1}^{k+2}$. Puisque $Q(1,0) Q(0,1) \neq 0$, la décomposition canonique de $\widetilde{Q}(X, Y)=X Y Q(X, Y)$ s'écrit, avec la notation (1·3),

$$
\widetilde{Q}=\prod_{1 \leqslant h \leqslant r} R_{h}^{\gamma_{h}} R_{r+1} R_{r+2}
$$

avec $R_{r+1}(X, Y):=X$ et $R_{r+2}(X, Y):=Y$. Posant $\widetilde{\boldsymbol{R}}:=\left\{R_{h}\right\}_{h=1}^{r+2} \in \mathbb{Z}[X, Y]^{r+2}$, nous avons, avec la notation $(1 \cdot 10)$,

$$
E_{\widetilde{\boldsymbol{R}}}(u+x) \asymp E_{\widetilde{R}}(\min \{u, x\})=E_{\boldsymbol{R}}(\min \{u, x\}) \asymp E_{\boldsymbol{R}}(u+x) .
$$

De plus

$$
\varrho_{\widetilde{Q}}^{+}(p)= \begin{cases}\varrho_{Q}^{+}(p)+2 p-1 & \text { si } p \nmid Q(1,0) Q(0,1) \\ \varrho_{Q}^{+}(p)+p-1 & \text { si } p \mid Q(1,0) Q(0,1), p \nmid(Q(1,0), Q(0,1)) \\ \varrho_{Q}^{+}(p) & \text { si } p \mid(Q(1,0), Q(0,1)) .\end{cases}
$$

Il suit

$$
\prod_{g<p \leqslant x}\left(1-\frac{\varrho_{\widetilde{Q}}^{+}(p)}{p^{2}}\right) \asymp \frac{Q(1,0) Q(0,1)}{\varphi(Q(1,0)) \varphi(Q(0,1))(\log x)^{2}} \prod_{g<p \leqslant x}\left(1-\frac{\varrho_{Q}^{+}(p)}{p^{2}}\right),
$$

ce qui fournit la conclusion souhaitée.

\section{Bibliographie}

[1] R. de la Bretèche \& T.D. Browning, Sums of arithmetic functions over values of binary forms, Acta Arithmetica $\mathbf{1 2 5} \mathrm{n}^{\circ} 3$ (2006), 291-304.

[2] R. de la Bretèche \& T.D. Browning, Binary linear forms as sums of two squares, Compos. Math., 144 (6), (2008), 1375-1402. 
[3] R. de la Bretèche \& T.D. Browning, Le problème des diviseurs pour des formes binaires de degré 4, J. reine angew. Math., 646, (2010), 1-44.

[4] R. de la Bretèche \& T.D. Browning, Binary forms of two squares and Châtelet surfaces, Israel J. Math., à paraître.

[5] R. de la Bretèche, T.D. Browning \& E. Peyre, On Manin's conjecture for a family of Châtelet surfaces, Ann. of Math., à paraître.

[6] R. de la Bretèche \& G. Tenenbaum, Oscillations localisées sur les diviseurs, prépublication (2011).

[7] R. de la Bretèche \& G. Tenenbaum, Sur la conjecture de Manin pour certaines surfaces de Châtelet, prépublication (2011).

[8] T.D. Browning, Linear growth for Châtelet surfaces, Math. Annalen 346 (2010), 41-50.

[9] S. Daniel, On the divisor-sum problem for binary forms, J. reine angew. Math. 507 (1999), $107-129$.

[10] S. Daniel, Uniform bounds for short sums of certain arithmetic functions of polynomial arguments, manuscrit non publié (2000).

[11] R.R. Hall and G. Tenenbaum, Divisors, Cambridge tracts in mathematics 90, Cambridge University Press (1988, paperback ed. 2008).

[12] K. Henriot, Nair-Tenenbaum bounds uniform with respect to the discriminant, prépublication (2009).

[13] C. Hooley, A new technique and its applications to the theory of numbers, Proc. London Math. Soc. (3) 38 (1979), 115-151.

[14] H. Maier \& G. Tenenbaum, On the normal concentration of divisors, 2, Math. Proc. Camb. Phil. Soc. $147 \mathrm{n}^{\circ} 3$ (2009), 593-614.

[15] T. Nagell, Introduction to number theory. 2nd ed., Chelsea, 1964.

[16] M. Nair, Multiplicative functions of polynomial values in short intervals, Acta Arith. 62 (1992), $257-269$.

[17] M. Nair \& G. Tenenbaum, Short sums of certain arithmetic functions, Acta Math. 180, (1998), 119-144.

[18] H. Maier and G. Tenenbaum, On the normal concentration of divisors, J. London Math. Soc. (2) 31 (1985), 393-400.

[19] E. Peyre, Hauteurs et mesures de Tamagawa sur les variétés de Fano, Duke Math. J. 79 (1995), 101-218.

[20] P. Shiu, A Brun-Titchmarsh theorem for multiplicative functions, J. reine angew. Math. 313 (1980), 161-170.

[21] C.L. Stewart, On the number of solutions of polynomial congruences and Thue equations. $J$. Amer. Math. Soc. 4 (1991), 793-835.

[22] G. Tenenbaum, Sur la concentration moyenne des diviseurs, Comment. Math. Helvetici 60 (1985), 411-428.

[23] G. Tenenbaum, Introduction à la théorie analytique et probabiliste des nombres, troisième édition, coll. Échelles, Belin, 2008, 592 pp.

Régis de la Bretèche

Institut de Mathématiques de Jussieu

UMR 7586

Université Paris Diderot-Paris 7

UFR de Mathématiques, case 7012

Bâtiment Chevaleret

75205 Paris Cedex 13

France

breteche@math.jussieu.fr
Gérald Tenenbaum

Institut Élie Cartan

Université de Nancy 1

BP 239

54506 Vandœuvre Cedex

France

gerald.tenenbaum@iecn.u-nancy.fr 